\title{
The Acute and Delayed Effects of Vagal Nerve Stimulation on an Absence Epilepsy Model in WAG/Rij
}

\author{
Ramazan UYAR ${ }^{1}$, Cezmi Cagri TURK ${ }^{1}$, Nejat ISIK², Mufit KALELIOGLU ${ }^{3}$, Filiz ONAT ${ }^{4}$, Memet OZEK ${ }^{3}$ \\ ${ }^{1}$ Antalya Education and Research Hospital, Department of Neurosurgery, Antalya, Turkey \\ 2istanbul Göztepe Education and Research Hospital, Department of Neurosurgery, İstanbul, Turkey \\ ${ }^{3}$ Acıbadem University, School of Medicine, Department of Neurosurgery, İstanbul, Turkey \\ ${ }^{4}$ Marmara University, School of Medicine, Department of Pharmacology, İstanbul, Turkey
}

\section{ABSTRACT}

AIM: Vagal nerve stimulation (VNS) is an effective method of treatment for epilepsy patients either unresponsive to medical therapy or not suitable for resective surgeries. We designed an experimental study on Wistar Albino Glaxo rats from Rijswijk (WAG\Rij) to investigate the effects of VNS on a non-convulsive epilepsy model.

MATERIAL and METHODS: The experiment was performed on six WAG/Rij rats, a validated strain for genetic absence seizures. The animals were underwent VNS and the effects were investigated on electroencephalography (EEG) recordings at 22, 24, 26 hours of stimulation and 15 days after the cessation, for duration of spike and wave complexes (SWC), the numbers, mean duration of SWC and frequencies in an hour.

RESULTS: EEG recordings demonstrated that the mean duration of SWC was 353.1 seconds and the number of activity per hour was 62 at the baseline. There were statistically significant decreases in the total duration of SWC and the number of activities (61.8\% and $78 \%$ decrease, respectively). There were no significant decreases in the mean duration of SWC and the frequencies.

CONCLUSION: The acute stimulation of the vagal nerve caused a statistically significant decrease both in overall duration of SWC and the number of complexes in an hour. Moreover, the positive effects seemed to last even 15 days after the cessation of the stimulation. Further studies focusing on different stimulation parameters and delayed effects of the VNS on human absence seizures are warranted.

KEYWORDS: Absence, Animal model, Epilepsy, Vagal nerve stimulation, WAG/Rij rat

\section{INTRODUCTION}

Vagal nerve stimulation (VNS) is an effective method of treatment for patients with epilepsy either unresponsive to medical therapy or not suitable for resective surgeries. A decrease in seizure frequency was shown in the literature with consensus but the exact mechanism of action and the particular types of responsive epilepsy (e.g. convulsive or non-convulsive) are still obscure $(2,4,11)$.

Absence seizures are a type of non-convulsive generalized epilepsy. They typically present with abrupt cessation of an on- going activity and transient unresponsiveness to surrounding stimuli. The patients generally have a good outcome and high success rates can be achieved with medical therapy.

As VNS is generally reserved for medically intractable or drug intolerant patients, effects of the stimulation for absence seizures is not well studied. However, knowing the efficacy and characteristics of VNS on generalized non-convulsive epilepsy, e.g. absence seizures, will potentially enlighten the mechanisms and extent of VNS actions on brain activities (5). 
Some previous studies focused on this subject in an animal model, with Genetic Absence Epilepsy Rats from Strasbourg (GAERS) $(4,5)$. We designed an experiment on Wistar Albino Glaxo rats from Rijswijk (WAG\Rij). There are basically two genetic absence epilepsy models in rats; GAERS and WAGVRij. On the other hand, both strains have distinct EEG characteristics. The present study concentrated on the effects of VNS on a non-convulsive epilepsy model.

\section{MATERIAL and METHODS}

\section{Animals}

There are basically two inbred rats well-validated for absence epilepsy models; WAG/Rij and GAERS. Both strains demonstrate spontaneously occurring seizures and the EEG findings are typical with Spike and Wave Complexes (SWC), similar to those in human absence epilepsy. For the present study, six male WAG\Rij rats, weighting 240-320 gram and aged 6-7 months were used. The animals were provided by the Department of Physiology at Marmara University, İstanbul. All animals were treated according to guidelines approved by the European Ethics Committee. The study protocol was approved by the Experimental Animal Ethics Committee of Marmara University, School of Medicine.

\section{Anesthetic and Surgical Procedures}

The WAG $\backslash$ Rij animals were anesthetized with a combination of ketamine (50 $\mathrm{mg} \backslash \mathrm{kg})$ and chlorpromazine $(0.75 \mathrm{mg} \backslash \mathrm{kg})$ by intraperitoneal injections. The first step of the surgery was performed on the Rat Stoelting Stereotactic frame (Stoelting Biological Research Apparatus 21025 Comerio, Varese, Italy). The frame was equipped with 3-dimensional micrometers and 2 electrode holders. The frame was positioned in the rat's mouth and both external acoustic meatuses stabilized by two rod-fixators (Figure 1B).

The animals were shaved and a vertical incision was performed over the scalp. The coordinates for bipolar EEG recording were determined by Rat's Brain Stereotactic atlas of Paxinos and Watson (10), with the bregma being the main point. The burr holes were drilled according to these measurements.

Bipolar recording electrodes were implemented through 4 burr holes, bilaterally located in the frontal and parietal regions. The electrodes were fixed together with cuff-electrode leads on the skull with acrylic (Figures 1A, B).

The rats were awakened and moved to their cage, staying for 3 days for the recovery period. Basal EEG recordings were obtained by the polygraph system of Grass Models (Grass Instruments, USA). All rats had absence seizures and their EEG recordings were obtained. Spike and Wave complexes (SWC) were determined as described by Akman et al (1); trains of sharp and asymmetrical spikes, which are followed by slow waves lasting 1 second or more. The onset and cessation of this complex was identified by a higher amplitude (at least two-fold) compared to baseline values. Cumulative total duration of the complexes and numbers were measured for 1 hour. The mean duration of the complexes was calculated by dividing the total duration by the number of seizures. All recordings were performed during the rat's normal activities.

After basal EEG recordings were obtained, the rats were anesthetized once more. They are positioned supine on the surgical table and prepared for left vagal nerve exposure. A skin incision was followed by dissection medial to the sternocleidomastoid muscle towards the carotid sheath with microsurgical techniques (Karl Kaps D6334, Asslar, Germany). The vagus nerve was exposed. VNS was implemented to the left vagus nerve (Neurocybernetic Prosthesis (NCP), Cybernetics Inc., Webster, TX, USA). The impedance of the electrode-to-vagus nerve interface was measured and was found to be around 1-3 kilo-ohms. The electrode line was externalized at the back of neck and the procedure was completed (Figure 2). The stimulator was arranged with the parameters in Table I.

\section{Experiment}

The animals were allowed to recover for 1 day before the experiments were started. They were placed in the recording

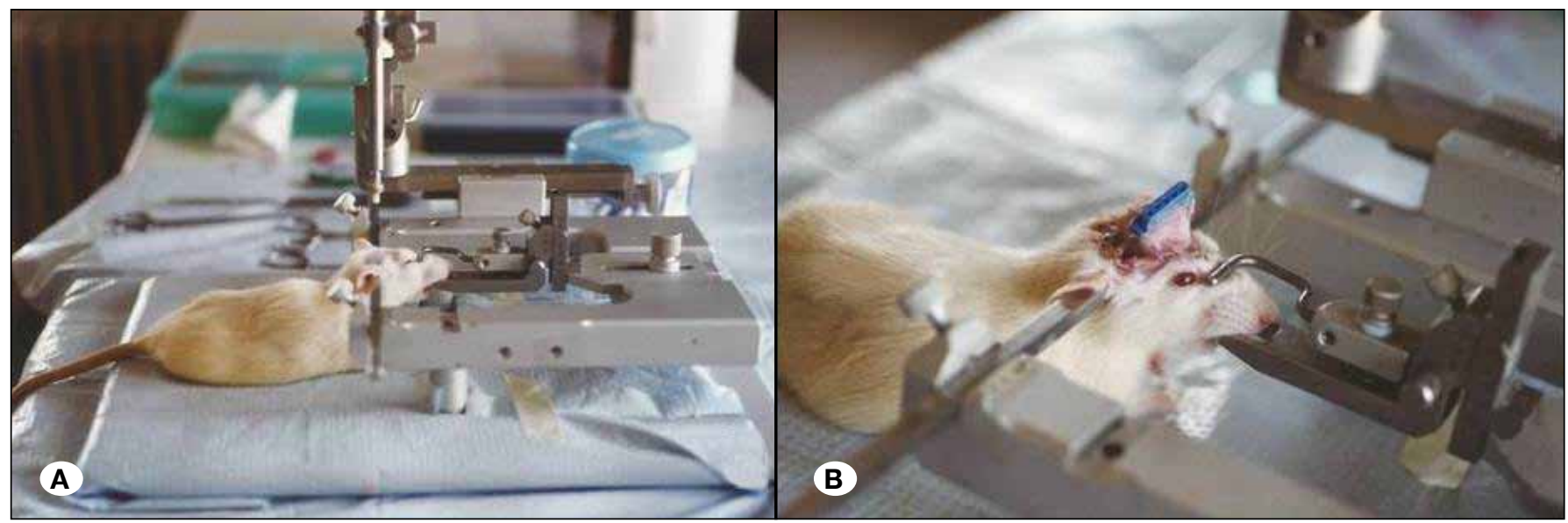

Figure 1:Images demonstrating experiment, (A) rats positioning on the frame, (B) after the connection of electrodes to cranium by acrylic. 
chambers through the whole experimental period. The experiments were performed after 22, 24 and 26 hours after the procedures during the morning and afternoon hours and the $15^{\text {th }}$ postoperative day after the vagal nerve stimulator was removed (Figure 2). During the experiments, the animals were freely moving and fed ad libitum.

The EEG recordings were performed for each of the 6 animals separately for 1 hour and the numbers and durations of SWC, mean seizure durations and frequencies were documented.

Statistical analysis was performed. The EEG recordings and their parameters at basal, $22^{\text {nd }}, 24^{\text {th }}, 26^{\text {th }}$ hours and the recordings at the $15^{\text {th }}$ day after removal were evaluated by using the ANOVA test for Friedman's non-parametric repeated measures.

Table I: The Parameters for the Vagal Nerve Stimulator

\begin{tabular}{lc}
\hline Electric Current & $\mathbf{1}$ milliampere \\
\hline Frequency & 30 hertz \\
\hline Pulse Width & 500 microseconds \\
\hline Time-on & 30 seconds \\
\hline Time-off & 10 minutes \\
\hline
\end{tabular}

\section{RESULTS}

For each rat, SWC were determined in one-hour EEG recordings (Figure 2). The mean durations of SWC for basal recordings and after the stimulations are presented at Figure 3. The cumulative duration of seizures were 353.1 seconds at first, at a mean number of $62 \mathrm{SWC/hour} \mathrm{(Figure} \mathrm{3).} \mathrm{The}$ measurements for vagal nerve stimulations at hours 22, 24, 26 and 15 days after the discontinuation are presented in the Table II.

The comparison between the basal EEG measurements and those after VNS showed that the mean duration of seizure activities in one hour declined from 353.1 with a steady improvement to 63.1 at 26 hours (61.8\% improvement). This figure continued at the recordings after 15 day of stimulations, being 68 seconds. Statistically, this difference between basal EEG recordings and measurements at hours 22, 24, 26 and at 15 days indicated a significant decrease in seizure activities $(\mathrm{P}<0.05)$.

Similar results were also obtained regarding the number of SWC in one-hour recordings. There was a steady trend for declining number of SWC at 22, 24, 26 hours; the least number of SWC was 13.5, reached at 26 hour recordings (mean 62 at baseline), and then a slight increase was observed at 15 day recordings (mean 15). These decreases in the number of

Table II: The Table Demonstrates the Mean Values for Cumulative Duration of Spike and Wave Complexes (SWC), the Number Per Hour, Mean Duration of SWCS and Means for Frequency Before and After the Procedure at Particular Times. The Difference is Statistically Significant $(p<0.05)$.

\begin{tabular}{|c|c|c|c|c|c|}
\hline EEG Findings/hour & Baseline & at $22^{\text {nd }}$ hour & at $24^{\text {th }}$ hour & at $26^{\text {th }}$ hour & after 15 days \\
\hline Mean of cumulative SWC durations (seconds) & 353.1 & 87.7 & 95.1 & 63.1 & 68 \\
\hline Mean number of SWC & 62 & 18 & 14.8 & 13.5 & 15 \\
\hline Mean duration of SWC (seconds) & 5.9 & 64.5 & 4.9 & 4.4 & 48.6 \\
\hline Mean frequency $(\mathrm{Hz})$ & 8.65 & 8.6 & 8.6 & 8.6 & 8 \\
\hline
\end{tabular}

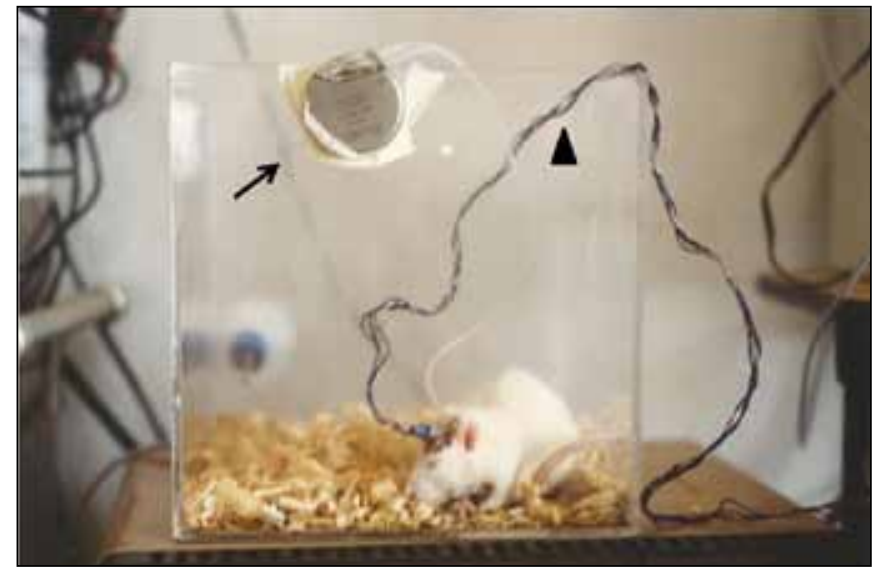

Figure 2: Images after implementation of VNS, rats placed in plexiglass cages. Both the VNS (arrow) and EEG recording electrodes (arrowhead) can be seen.

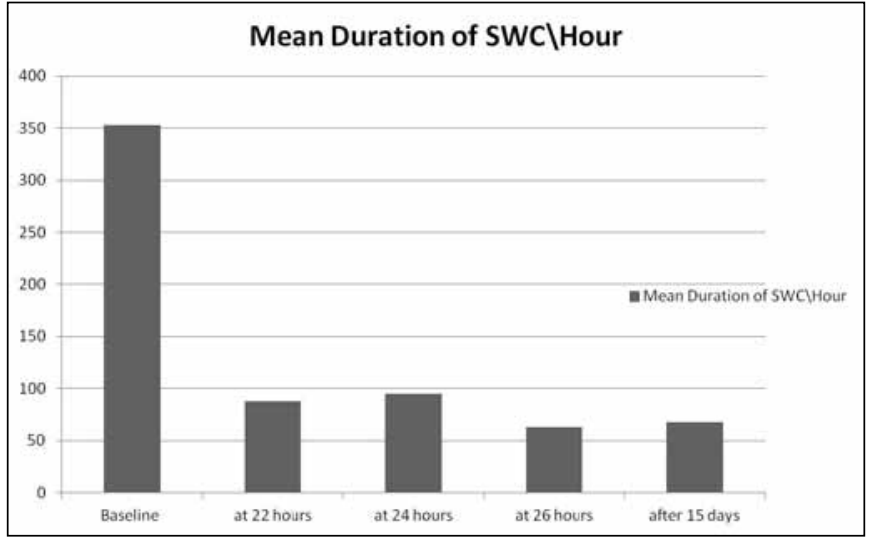

Figure 3: Image shows the comparative bar for mean durations of spike and wave complexes from baseline values of hours $22^{\text {nd }}$, $24^{\text {th }}$ and $26^{\text {th }}$ of stimulation and 15 days after the cessation of the stimulation. This demonstrates significant decrease in the epileptic activity. 


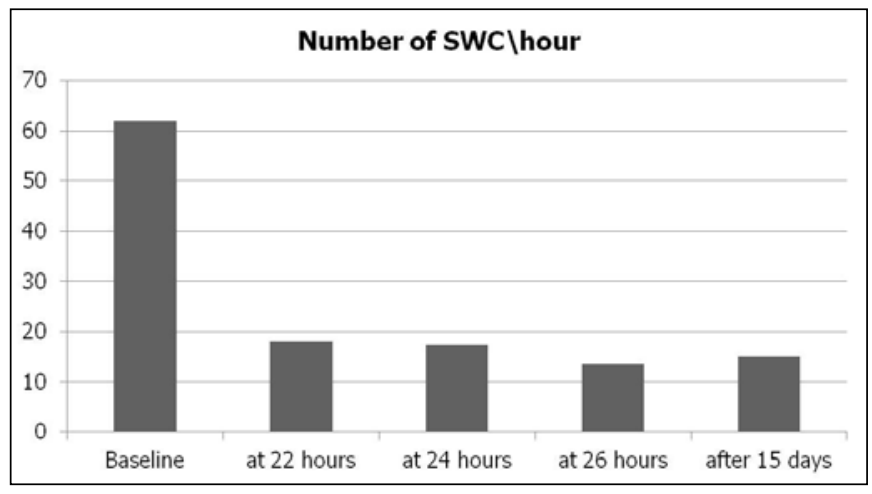

Figure 4: Image shows the comparative bar for number of spike and wave complexes observed in one hour, the values from baseline and at $22^{\text {nd }}, 24^{\text {th }}, 26^{\text {th }}$ hours of stimulation and 15 days after the cessation of the stimulation.

SWC was also statistically significant $(P<0.05)$ compared to baseline (Figure 4).

There were two parameters where no statistically significant difference was observed; the mean duration of seizures per hour and mean frequencies at EEGs.

\section{DISCUSSION}

The exact mechanism of action for VNS is still obscure and understanding the underlying process will help define the specific epilepsy syndromes that could be managed by this approach. The literature generally relies on experiments and experiences with convulsive seizures, while non-convulsive cases, e.g. absence seizures, progress by different neuronal mechanisms (6).

Absence seizures in humans are claimed to have a genetic predisposition without a structural abnormality. They are generally associated with bilateral and synchronous SWC on EEG recordings (7). Absence seizures are generally accepted to have a benign temper and there is therefore paucity in the literature on the efficacy of VNS in this type of non-convulsive seizures.

There are some reports by Dedeurwaerdere et al. who particularly studied the acute and chronic effects of VNS on GAERS rats, one of the two animal strains validated for absence seizures $(4,5)$. In the article on acute effects of VNS; they could not show any effect of applying stimulation to vagal nerve on SWC whether performed at the onset or during the discharges. They concluded that as the epileptogenesis took months to develop in GAERS, it would be wise to apply stimulation in chronic bases to observe any difference in the recordings (4). One year later, chronic effects were studied and published by Dedeurwaerdere et al.; this time the cumulative duration of spike and wave discharges was shown to decrease significantly, but still could not reach statistically significant levels (5).

Based on these data, the secondary observations and findings on WAG $\backslash$ Rij rats gains importance, as this is an equally accepted model for absence seizures. In fact, there are re- ports stating important differences between these two strains regarding EEG characteristic (probably representing different absence seizure types) (1).

In the following model, acute effects and delayed effect of the VNS on WAG/Rij were observed. After the onset of the stimulation, an abrupt and statistically significant $(p<0.05)$ decrease was observed in the cumulative duration of SWCs. Moreover the significance was also valid for the total number of seizures per hour. The clinical alertness was higher after VNS application, too. These values were improved at hours 22, 24 and 26. These data were different than those observed in previous studies $(4,5)$. This difference may come from variations between the animal strains, per se.

There are reported differences between GAERs and WAG/ Rij strains such as the age of onset of SWC, localization of epileptogenic focuses, distribution of some receptors (e.g. dopamine), and affects of kindling processes $(1,8)$. Sitnikova and Van Luijtelaar emphasized the presence of two different origins of SWC in WAG/Rij (compared to one in GAERS); one form being generalized discharges spreading through the cortex with similarities to humans both clinically and electrophysiologically (12). Moreover, GAERS were reported to have a more wide-spread involvement of the white matter tracts compared to WAGIRij strains. The higher numbers and severity of SWC in GAERS were correlated with these findings (3).

In humans, the effects of VNS were observed over longer periods, meaning a cumulative effect. In some previous studies, the efficacy of VNS was also questioned in terms of the duration of epileptogenesis before treatment and the duration of VNS applications. Interestingly, our study indicated a more prolonged benefit, even after the stimulation had been ceased. The overall total duration of SWC decreased and remained so after 15 days of stimulation (353 second baseline to 63 at 26 hours and 68 after 15 days). These figures may raise curiosity about the probable effects of VNS for absence seizures in humans.

There are some electrophysiological differences between humans and animal models for absence epilepsy; in humans, SWC repeats at slower frequencies (approximately $3 \mathrm{~Hz}$ ) whilst in the epileptic rats this figure is reported around to be $7-11 \mathrm{~Hz}$ (9). There are also some conceptual and electrophysiological differences in absence seizures compared to convulsive ones. Because of these, the effects of VNS on absence seizures needs to be investigated in humans, to better understand the underlying mechanism of actions.

\section{CONCLUSION}

This study demonstrated the effects of VNS on an animal model of a genetically epileptic rat strain: WAG/Rij. The acute stimulation of the vagal nerve caused a statistically significant decrease in both the overall duration of SWC and the number of complexes in an hour. Moreover, the positive effects seemed to last even 15 days after cessation of the stimulation. Further studies focusing on different stimulation parameters and delayed effects of the treatment on human absence seizures are warranted. 


\section{REFERENCES}

1. Akman O, Demiralp T, Ateş N, Onay FY: Electroencephalographic differences between WAG/Rij and GAERS rat models of absence epilepsy. Epilepsy Res 89:185-93, 2010

2. Ben-Menachem E: Vagus-nerve stimulation for the treatment of epilepsy. Lancet Neurol 1: 477-82, 2002

3. Chahboune H, Mishra AM, SeSalvo MN, Staib LH, Purcaro M, Scheinost D, Papademetris X, Fyson SJ, Lorincz ML, Crunelli $\mathrm{V}$, Hyder F, Blumenfeld $\mathrm{H}$ : DTI abnormalities in anterior corpus callosum of rats with spike-wave epilepsy. Neuroimage 47: 459-66, 2009

4. Dedeurwaerdere S, Vonck K, Claeys P, Van Hese P, D'Have M, Grisar T, Naritoku D, Boon P: Acute vagus nerve stimulation does not suppress spike and wave discharges in genetic absence epilepsy rats from Strasbourg. Epilepsy Res 59:191198, 2004

5. Dedeurwaerdere S, Vonck K, Van Hese P, Wadman W, Boon $P$ : The acute and chronic effect of vagus nerve stimulation in genetic absence epilepsy rats from Strasbourg (GAERS). Epilepsia 46: 94-97, 2005
6. Eskazan E, Onat FY, Aker R, Oner G: Resistance to propagation of amygdaloid kindling seizures in rats with genetic absence epilepsy. Epilepsia 43: 1115-1119, 2002

7. Niedermeyer E: Primary (idiopathic) generalized epilepsy and underlying mechanisms. Clin Electroencephalogr 27:1-21, 1996

8. Onat FY, Aker RG, Gurbanova AA, Ateş N, van Luijtelaar G: The effect of generalized absence seizures on the progression of kindling in the rat. Epilepsia 48:150-156, 2007

9. Panayiotopoulos, CP: Typical absence seizures and related epileptic syndromes: Assessment of current state and directions for future research. Epilepsia 49: 2131-2139, 2008

10. Paxinos G, Watson G: The Rat Brain in Stereotaxis Coordinates. California: Academic Press, 1997

11. Rutecki P: Anatomical, physiological and theoretical basis for the antiepileptic effect of vagus nerve stimulation. Epilepsia 31:1-6, 1990

12. Sitnikova E, Van Luijtelaar G: Electroencephalographic characterization of spike-wave discharges in cortex and thalamus in WAG/Rij rats. Epilepsia 48: 2296-2311, 2007 